\title{
Panic Disorder and Serotonin Reuptake Inhibitors Predict Coupling of Cortical and Cardiac Activity
}

\author{
Erik M Mueller*,', Christian Panitz', Yvonne Nestoriuc', Gerhard Stemmler' and Jan Wacker' \\ 'Department of Psychology, Philipps-Universität Marburg, Marburg, Germany
}

\begin{abstract}
Panic attacks, the cardinal symptom of panic disorder (PD), are characterized by intense physiological reactions including accelerated heart activity. Although cortical processes are thought to trigger and potentiate panic attacks, it is unknown whether individuals with PD have a general tendency to show elevated cortico-cardiac interactions, which could predispose them for brain-driven modulations of heart activity during panic. Consistent with this hypothesis, serotonin, a highly relevant neurotransmitter for panic and PD presumably affects the cortical control of the heart. The current study thus aimed to test whether PD and serotonin reuptake inhibitor (SRI) intake are related to corticocardiac interactions in the absence of acute panic. Human participants with PD $(n=22)$, major depression (MD, clinical control group, $n=2 \mathrm{I}$ ) or no psychiatric diagnosis (healthy control group, $n=23$ ) performed a gambling task. To measure cortico-cardiac coupling, the within-subject covariation of single-trial EEG after feedback presentation and subsequent changes in heart period was determined. As in prior studies, there was a significant time-lagged covariation of EEG and heart activity indicating that trial-by-trial fluctuations of feedback-evoked EEG amplitude determined how much heart activity accelerated seconds later. Importantly, this covariation pattern was significantly potentiated in PD vs control participants. Moreover, concurrent SRI intake further augmented brain-heart covariation in individuals with PD and MD. The present findings demonstrate that PD and serotonin are associated with altered brain-heart interactions in a non-panic situation. Future work should clarify whether brain-heart coupling has a causal role in PD, for example by facilitating panic attacks.

Neuropsychopharmacology (20I4) 39, 507-5I4; doi:I0.1038/npp.2013.224; published online 25 September 2013
\end{abstract}

Keywords: heart rate; P300; brain-heart coupling; depression; feedback

\section{INTRODUCTION}

Individuals diagnosed with panic disorder (PD), one of the most severe anxiety disorders, suffer from unexpected panic attacks: sudden symptoms of intense perceived physiologi$\mathrm{cal}$ arousal and maladaptive interpretation thereof (eg, 'I'm having a heart attack!'). Although many models of panic focus on cognitive (Clark, 1986) or learning (Bouton et al, 2001) processes, high heritability estimates of PD (Kendler et al, 2001) further emphasize the importance of biological mechanisms (Gorman et al, 2000). As panic attacks are characterized by intensive peripheral physiological sensations with presumably neurogenic causes (Bouton et al, 2001; Clark, 1986; Gorman et al, 2000; Graeff and Del-Ben, 2008), PD may be related to potentiated neurotransmission between the central and peripheral nervous system. As cardiovascular sensations (ie, tachycardia, palpitations) are the most frequent peripheral manifestations of panic attacks (Barlow et al, 1985) the coupling of central and cardiac activity could be of particular relevance in PD (Friedman and Thayer, 1998). Because a number of studies indicate that objectively measured heart rate increases do not

\footnotetext{
*Correspondence: Dr EM Mueller, University of Giessen, Department of Psychology, Otto-Behaghel Str. IOF 39354, Giessen, Germany.

Tel: + 496419926 084, Fax: + 496419926099 ,

E-mail: erik.mueller@psychol.uni-giessen.de

Received 8 May 2013; revised 20 August 2013; accepted 22 August 2013; accepted article preview online 29 August 2013
}

necessarily occur during panic attacks (Margraf, 1990) nor differ between PD and healthy participants (Aronson et al 1989; Cowley et al, 1987; Gaffney et al, 1988), PD-related abnormalities may be identified within the interactions of the central and peripheral nervous system rather than at the level of cardiovascular responses per se.

Until recently, appropriate non-invasive methods to study the hypothesized role of central-peripheral nervous system interactions in PD were lacking. The purpose of the present study was to take advantage of a novel technique termed cardio-electroencephalographic covariance tracing (CECT; Mueller et al, 2010) to test for the first time whether brainheart coupling is disturbed in PD. The CECT method assesses time-lagged within-subject covariation of evoked single-trial EEG and heart period (HP) after an external stimulus. CECTs recently demonstrated that centromedial brain activity $300 \mathrm{~ms}$ after presentation of motivationally meaningful stimuli (eg, win/loss feedback in gambling tasks) predicts subsequent HP alterations (Mueller et al, 2012; Mueller et al, 2013; Mueller et al, 2010): the larger the EEG amplitude at this latency, the shorter the HP (ie, more acceleration) at subsequent beats. This phenomenon has been labeled ' $\mathrm{N} 300 \mathrm{H}$ ' to indicate a negative $(\mathrm{N})$ association between EEG amplitude at $300 \mathrm{~ms}$ and HP. The putative $\mathrm{N} 300 \mathrm{H}$ brain source is the anterior cingulate cortex (Panitz et al, 2013), a key region for central control of the viscera (Critchley et al, 2003). Consequently, we focus on $\mathrm{N} 300 \mathrm{H}$ for this initial test for disturbed brain-heart coupling in PD. 
Because the $\mathrm{N} 300 \mathrm{H}$ has been best validated for gambling tasks in which trial-by-trial feedback is given (Mueller et al, 2010; Panitz et al, 2013), we also used a gambling task in the present paradigm to evoke $\mathrm{N} 300 \mathrm{H}$.

$\mathrm{PD}$ is frequently accompanied by major depression (MD; Roy-Byrne et al, 2000), which is also linked to cardiovascular irregularities (Carney et al, 2001). Accordingly, altered brain-heart coupling in PD may be due to comorbid MD rather than reflecting a unique feature of PD. Thus, an MD clinical control group without a history of panic attacks was included in the current study to investigate the specificity of the hypothesized effects concerning PD.

Finally, there is evidence for a prominent role of serotonin (5-HT) in both, brain-heart communication (Jordan, 2005; Mueller et al, 2012; Mueller et al, 2013) and PD (Neumeister et al, 2004). Because serotonin reuptake inhibitors (SRIs) are commonly prescribed for PD and MD, we tested whether current SRI intake correlates with N300H. As both, pharmacological (Mueller et al, 2012) and genetically driven reductions of 5-HT signaling (David et al, 2005; Mueller et al, 2013), attenuate $\mathrm{N} 300 \mathrm{H}$, we expected that SRIs, by increasing 5-HT availability should elevate $\mathrm{N} 300 \mathrm{H}$.

\section{MATERIALS AND METHODS}

\section{Participants}

A total of $n=66$ participants were recruited through advertisements, posters, or by referral from the department's outpatient ambulance. Diagnosis was assessed using a standardized clinical interview based on DSM-IV and ICD-10 criteria (Margraf, 1994), and inclusion criteria for the control, $\mathrm{MD}$, and $\mathrm{PD}$ groups, respectively, were (A) no lifetime DSM-IV diagnosis, (B) a current DSM-IV diagnosis of mild, moderate or severe major depressive episode without any history of panic attacks or (C) a DSM-IV diagnosis of current PD with or without agoraphobia (comorbid depression allowed for the PD group). General inclusion criteria were age above 18, BMI between 17 and 30 , and the absence of psychotic, bipolar, neurological, or cardiovascular disorders. Concurrent use of prescription drugs was assessed to probe relationships between concurrent SRIs intake and cortico-cardiac coupling. For the control, MD, and PD groups, the final sample consisted of $n=23,21$, and 22 participants ( $n=12$ with agoraphobia), respectively. For various sample characteristics see Table 1.

\section{Procedure}

The study consisted of an interview and an experimental session, which were maximally 1 week apart. At the former, participants provided informed consent before the clinical interview was conducted. If inclusion/exclusion criteria were met, participants were given questionnaires to fill out at home including the Agoraphobic Cognitions Questionnaire (Chambless et al, 1984), the Beck Depression Inventory II (Beck et al, 1996), and the Anxiety Sensitivity Index 4 (Kemper, 2010).

An experimenter who was unaware of the participants' diagnosis conducted the experimental session. To minimize the influence of the (potentially agoraphobic) laboratory setting, calming information about the laboratory was provided for all participants and none of them reported any panic symptoms throughout the task. After a 10-min resting phase, participants performed a 15-trial test block and then the actual gambling task. At the end of the session, participants were debriefed and compensated with $35 €$ (46\$). The study protocol was approved by the Ethics Committee of the Psychology Department at Marburg University.

\section{Gambling Task}

Participants completed a 360-trial gambling task described in more detail elsewhere (Mueller et al, 2010; Sato et al, 2005). In each trial, participants could win or lose an initially indicated amount of money $(0,10$, or 50 cents) if they correctly guessed what kind of card the computer would draw. Positive (green circle), negative (red cross), or ambiguous (blue question mark) feedback was given after each trial indicating whether the initially displayed amount was won or lost in that trial. Participants did not know that the presentation of feedback types was quasi-randomized with balanced frequencies and they were told in advance that they could win between 10 and $15 €$ in total. In the end, however, each participant received $15 €$.

\section{Physiological Recording}

EEG was recorded at 64 channels with a sampling rate of $512 \mathrm{~Hz}$ using an Active Two system (BioSemi, Amsterdam, Netherlands), as reported in more detail elsewhere (Mueller et al, 2011). ECG was measured with three $\mathrm{Ag}-\mathrm{AgCl}$ flat electrodes in lead II configuration (right forearm, left leg, ground electrode on left arm) connected to the BioSemi system.

\section{Data Analysis}

EEG. EEG was re-referenced to linked mastoids, downsampled to $128 \mathrm{~Hz}$, highpass filtered $(1 \mathrm{~Hz})$ and manually screened for artifacts using EEGLAB (Delorme and Makeig, 2004) in MATLAB 7.5.0 (MathWork, Natick, Massachusetts, USA). Eye-blinks were removed using Independent Component Analysis. Data were segmented ( -200 to $781.25 \mathrm{~ms}$ relative to feedback) and baseline-corrected. These segments were used for CECTs as described below.

$H P$. R-spikes were automatically detected in the bandpass-filtered ECG $(1-30 \mathrm{~Hz})$ and HP traces were computed through a customized routine in MATLAB, which creates a continuous HP trace at $128 \mathrm{~Hz}$ where each sample reflects the distance in milliseconds between the preceding and succeeding R-spike. Resulting HP traces were manually screened for artifacts. Computed segments ranged from 0-5000 ms post stimulus and data were baseline-corrected by subtracting the value at stimulus onset. As with the EEG, single-trial data were used for CECTs.

CECTs. CECTs were computed as in prior studies (Mueller et al, 2012; Mueller et al, 2013; Mueller et al, 2010; Panitz et al, 2013). To allow CECT computation across the 350-420 trials available for analysis per individual after 
Table I Sample Characteristics of Patients with Panic Disorder, Major Depression, and Healthy Control Participants

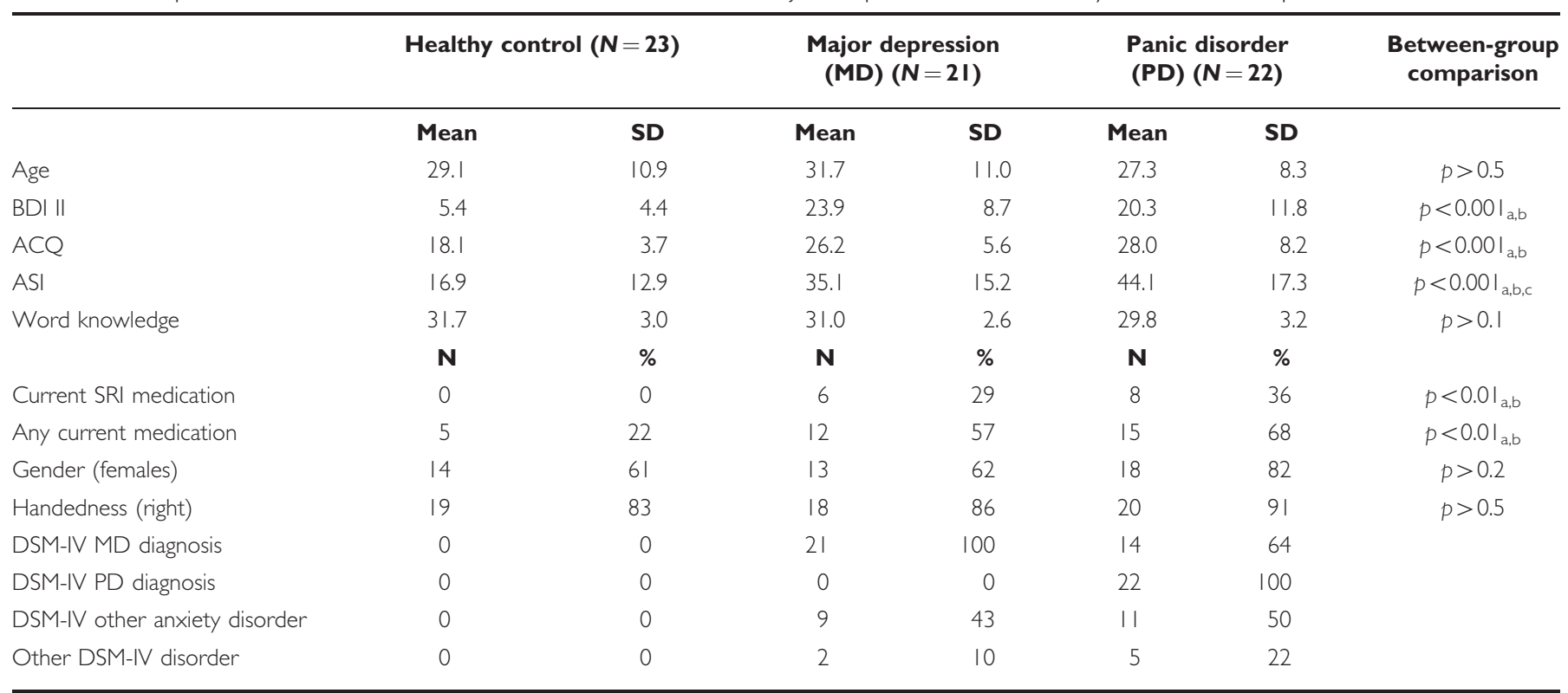

Abbreviations: ACQ, Agoraphobic Cognition Questionnaire; ASI, Anxiety Sensitivity Index; BDI II, Beck depression inventory II.

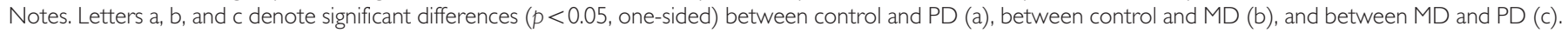

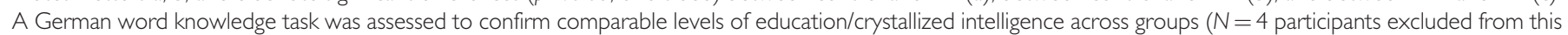
analysis due to immigration background or missing data). SRIs included citalopram, escitalopram, fluoxetine and venlafaxine. Any current medication included trimipramine, clomipramine, mirtazapine, thyroxine, beta-adrenergic blocking agents, opipramol, and contraceptives.

artifact screening, EEG segments were divided into 50 bins of $15.625 \mathrm{~ms}$ each, ranging from $0 \mathrm{~ms}$ (stimulus onset) to $781.25 \mathrm{~ms}$. HP segments were divided into 10 bins of $500 \mathrm{~ms}$, ranging from $0-5 \mathrm{~s}$. Next, CECTs were computed by correlating (Pearson's $r$ ) each EEG bin with each HP bin across trials within subjects, resulting in a $10 \times 50$ cross-correlation CECT matrix. CECT matrices were computed separately for each individual and feedback valence. Individual peak $\mathrm{N} 300 \mathrm{H}$ magnitudes were extracted as the most negative within-subject correlation of EEG from 250-500 ms and HP from 2-5s relative to the feedback stimulus. All $\mathrm{N} 300 \mathrm{H}$ values were Fisher transformed before statistical analysis.

Event-related potential and evoked cardiac response. To assess the event-related potential (ERP), the same segments that were used for the CECT analyses were averaged across trials, separately for each feedback valence (positive, negative, and neutral). For each ERP, the most positive value between 250 and $500 \mathrm{~ms}$ at channel $\mathrm{Cz}$ was measured, corresponding to the $\mathrm{N} 300 \mathrm{H}$ measurement latency in the EEG time domain and presumably reflecting the P300 (Nieuwenhuis et al, 2011).

Similarly, to assess the evoked cardiac response, segments were averaged across trials by feedback type. For each evoked cardiac response, the most negative HP value between 2 and $5 \mathrm{~s}$ was extracted, corresponding to the peak cardiac acceleration at the $\mathrm{N} 300 \mathrm{H}$ measurement latency in the HP time domain.

\section{Statistical Analyses}

To test for the effect of PD and MD on N300H, P300, and cardiac acceleration to feedback group (control vs PD vs
$\mathrm{MD}) \times$ valence (positive $v s$ negative $v s$ neutral feedback) ANOVAs were conducted with valence as a repeated measures factor.

\section{RESULTS}

\section{$\mathrm{N} 300 \mathrm{H}$}

General characteristics. As evident from the blue clusters in Figure 1, feedback-evoked EEG at $300 \mathrm{~ms}$ showed the expected negative covariation with subsequent HP thereby indicating robust feedback-evoked brain-heart coupling within individuals ('N300H'). The $\mathrm{N} 300 \mathrm{H}$ peak values had a mean EEG latency of $309 \mathrm{~ms}$ (SD: $36 \mathrm{~ms}$ ) and a mean HP latency of $3.4 \mathrm{~s}$ (SD: $1.0 \mathrm{~s}$ ). Spearman-Brown corrected splithalf reliability estimates (assessed by computing one peak $\mathrm{N} 300 \mathrm{H}$ for odd trials and one peak $\mathrm{N} 300 \mathrm{H}$ for even trials for each participant) revealed satisfactory reliability of the peak $\mathrm{N} 300 \mathrm{H}$ magnitude $(r=0.59)$. In support of the construct validity of $\mathrm{N} 300 \mathrm{H}$, individuals with higher $\mathrm{N} 300 \mathrm{H}$ values also showed increased cardiac acceleration after feedback $(r=0.26, p<0.04)$ and reported higher levels of arousal before the task $(r=0.31, p<0.02)$, indicating that $\mathrm{N} 300 \mathrm{H}$ is related to (but not redundant with) evoked cardiac responses and perceived arousal.

Relationships to PD and MD. The mean $\mathrm{N} 300 \mathrm{H}$ latencies did not differ between groups (EEG: $F_{(2,63)}=0.48, p>0.5$; HP: $\left.\quad F_{(2,} 6_{3)}=1.32, \quad p>0.2\right)$. Importantly, a feedback valence $\times$ group ANOVA revealed that $\mathrm{N} 300 \mathrm{H}$ magnitude significantly differed between groups $\left(F_{(2,} 63\right)=4.20$, $p<0.02$, see Figures $1 \mathrm{a}$ and 2 ) with higher magnitudes in $\mathrm{PD} v s$ control participants (Scheffé post hoc test PD vs control: $p<0.02)$. Depressed participants displayed 
a

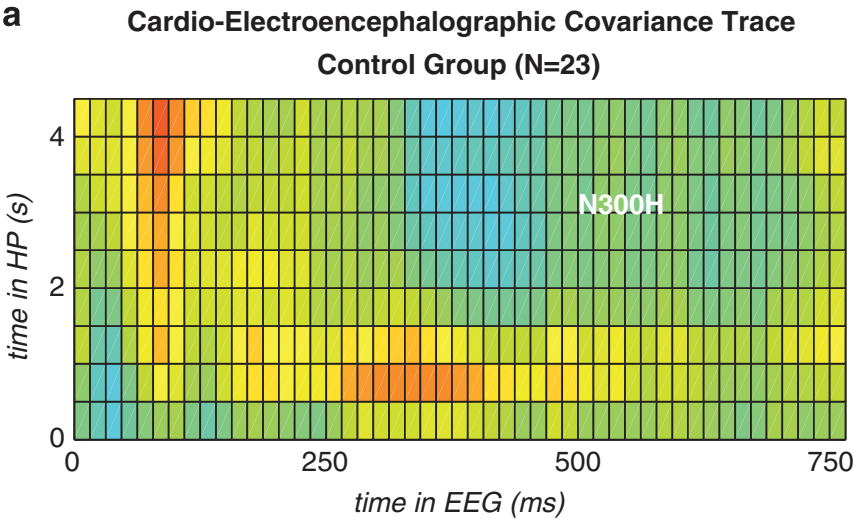

Depression Group ( $\mathrm{N}=\mathbf{2 1})$

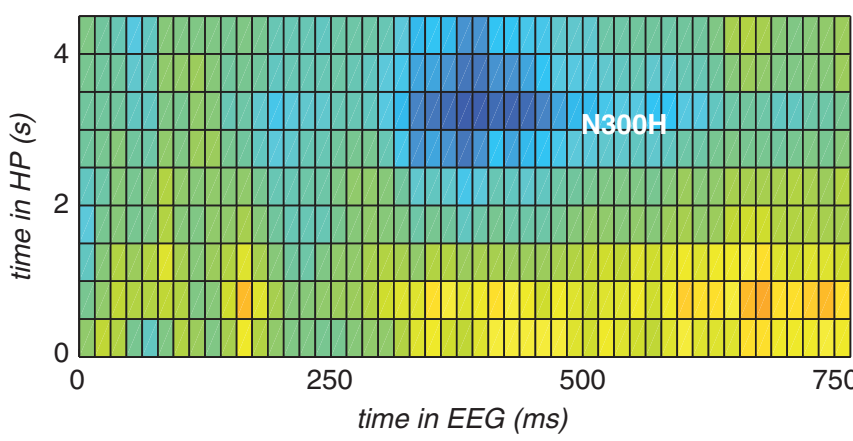

Panic Disorder Group (N=22)

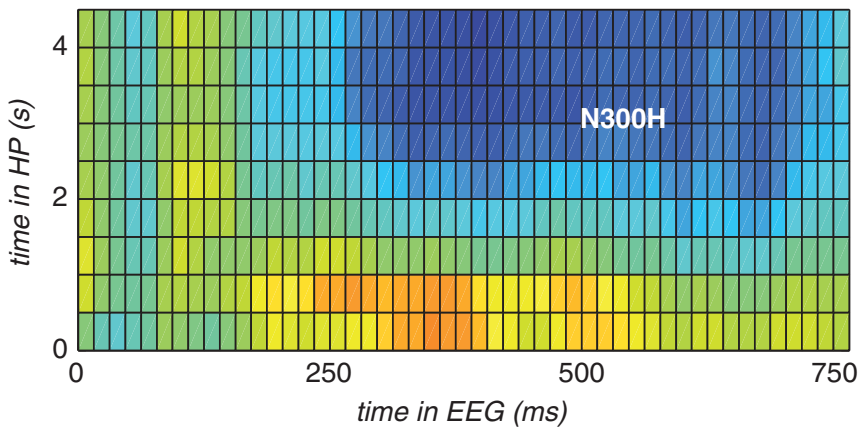

mean correlation $(r)$
0 b

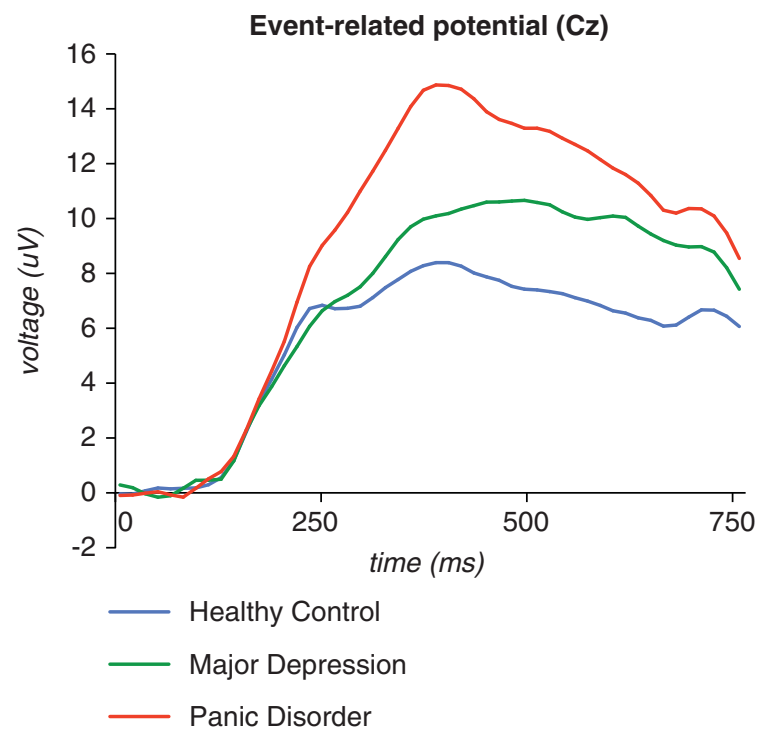

c
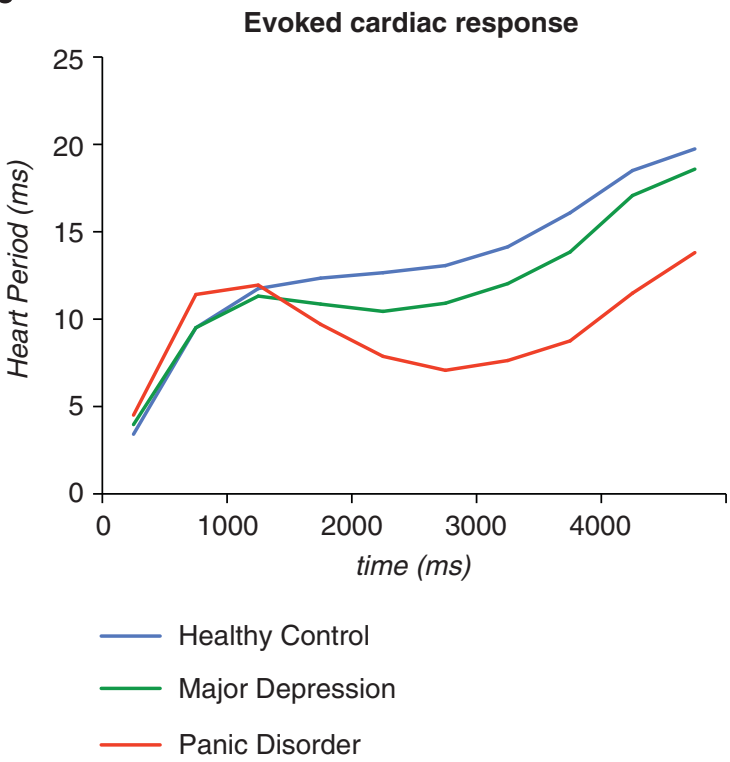

Figure I Panel a: Cardio-Electroencephalographic Covariance Traces for healthy control (top), major depression (MD, middle) and panic disorder (PD, bottom) participants at channel $\mathrm{Cz}$. Each box represents the group-average of within-subject correlations between feedback-evoked single-trial EEG and heart period (HP) as a function of lag in the EEG time domain ( $x$ axis) and HP time domain ( $y$ axis). Blue clusters around $300 \mathrm{~ms}$ in the EEG time domain and $2-5 s$ in the HP time domain indicate negative within-subject correlations between EEG at 300 ms and subsequent changes in HP (N300H). Feedbackevoked event-related potential at channel Cz (panel b) and modulations of HP (panel c) for healthy control (blue), MD (green) and PD (red) participants.

intermediate $\mathrm{N} 300 \mathrm{H}$ magnitudes, that neither differed from panic nor control participants $(p>0.2)$. There were no other main effects or interactions $(p>0.9)$.

Four control analyses confirmed that the larger $\mathrm{N} 300 \mathrm{H}$ in the PD vs control group was not driven by (i) comorbid depression, (ii) group differences in overall arousal, (iii) concurrent medication, or (iv) gender. First, when all participants with a diagnosis of MD were removed from the PD group, the difference between panic $(n=8)$ and control
( $n=23)$ remained significant $\left(t_{(29)}=2.83, p=0.01\right)$. Second, when self-reported arousal at the beginning of the procedure was partialled from $\mathrm{N} 300 \mathrm{H}$ ( $\left.r_{(\text {arousal, }} \mathrm{N} 300 \mathrm{H}\right)$ $=0.31, p<0.02)$, the difference between PD and control participants remained significant $\left(t_{(43)}=2.30, p<0.03\right)$. Third, when all participants currently taking any kind of medication were excluded from analysis, the difference between panic $(n=7)$ and control $(n=18)$ participants also remained significant $\left(t_{(23)}=2.26, p<0.04\right)$. Finally, when 


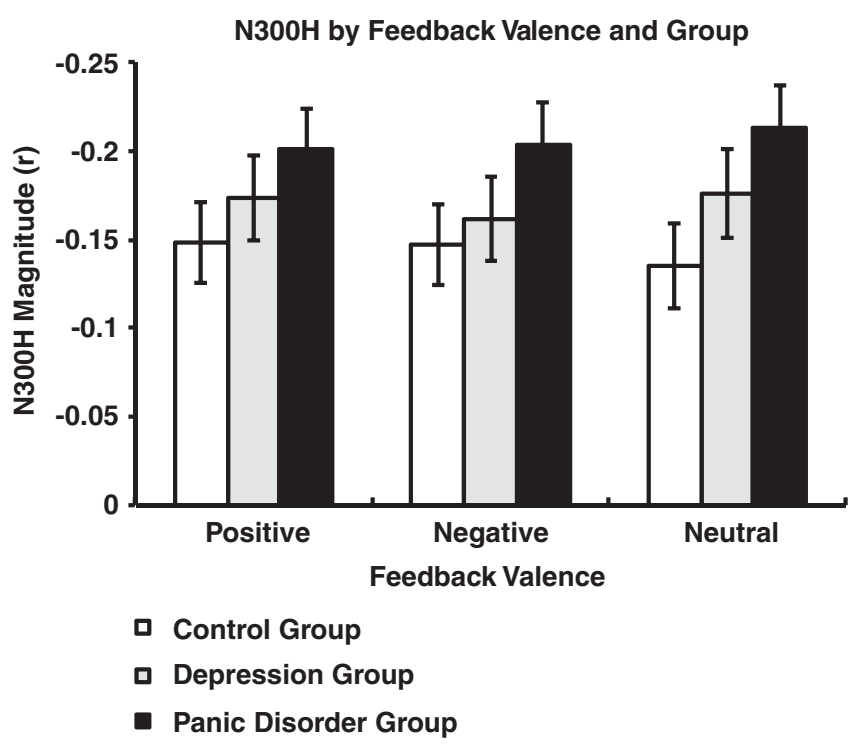

Figure 2 Barplot indicating mean (SEM) N300H magnitudes at channel $\mathrm{Cz}$ by group and feedback valence.

gender was included as a factor, the main effect for group likewise remained significant $\left(\mathrm{F}_{(1,60)}=5.60, p<0.01\right)$ and there were no significant interactions involving gender.

Temporal spread of N300H. Visual inspection of Figure 1 suggests that $\mathrm{N} 300 \mathrm{H}$ shows an extended temporal spread in PD (in addition to enhanced magnitude). Although not predicted a priori, we tested whether this effect was statistically significant by first counting for each individual the number of within-subject correlations exceeding $r \geqslant 0.08$ (corresponding to $p<0.05$ when correlating across 420 trials) in the individual CECT matrices. One subject (MD group) had a value more than three SD above the mean in this temporal spread variable and was therefore excluded from this analysis. Consistent with the visual impression, a nonparametric Kruskal-Wallis test indicated that groups differed significantly in temporal spread $\left(X_{(2)}=8.73, p<0.02\right)$ with a significant difference between the control and $\mathrm{PD}\left(X_{(1)}=8.0\right.$, $p<0.005)$, between $\mathrm{PD}$ and $\mathrm{MD}\left(X_{(1)}=4.59, p<0.05\right)$ but not between control and MD group $(p>0.5)$.

Concurrent SRI intake. As shown in Figure 3, individuals with PD or MD who were treated with SRIs at the time of the investigation $(n=14)$ showed significantly larger $\mathrm{N} 300 \mathrm{H}$ magnitudes (across different feedback types: $t_{(41)}=2.60$, $p<0.015)$ and a trend for increased temporal spread of $\mathrm{N} 300 \mathrm{H}\left(t_{(41)}=1.95, p<0.06\right)$ than individuals with PD or MD who were not treated with SRIs $(n=29)$. Individuals currently taking SRIs did not show different values in mean questionnaire measures than non-medicated individuals with PD or MD ( $p$-values $>0.4)$.

\section{Event-Related Potential}

Similar to $\mathrm{N} 300 \mathrm{H}$, the $\mathrm{P} 300$ differed between groups $\left(F_{(2,63)}=5.12, p<0.01\right)$ and was significantly increased in $\mathrm{PD} v s$ control participants (Scheffé post hoc test for comparison of PD vs control: $p<0.01$, Figure 1). The MD

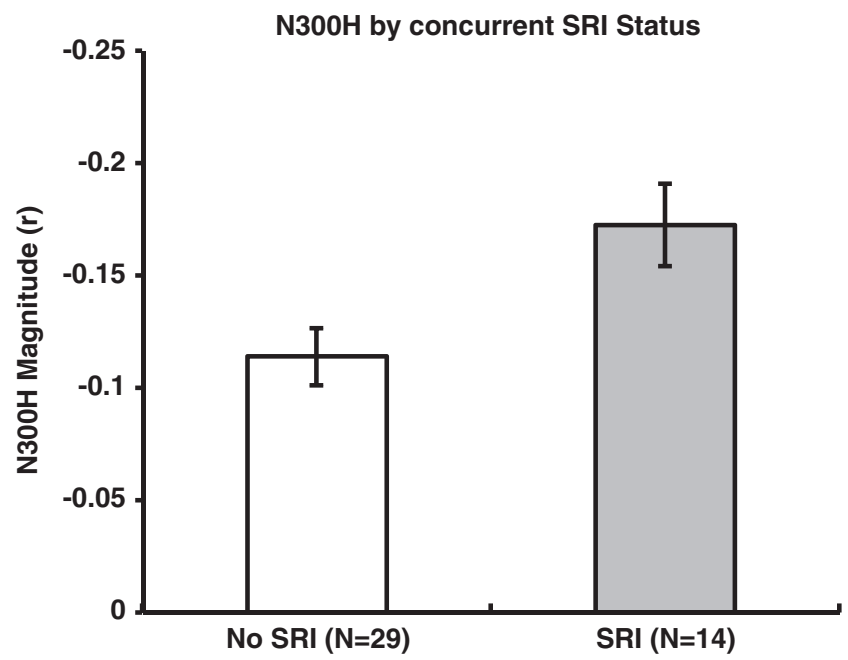

Figure 3 Barplot indicating mean (SEM) N300H magnitudes at channel $\mathrm{Cz}$ for individuals with or without SRI intake (clinical groups only).

group did not significantly differ from the other two groups $(p>0.1)$. There was also a main effect of feedback valence $\left(F_{(2,63)}=11.27, p<0.001\right)$ indicating that neutral feedback evoked a smaller amplitude than negative and positive feedback. When individuals currently taking any kind of medication were excluded from analysis, the difference between control $(n=18)$ and PD $(n=7)$ participants was marginally significant $\left(t_{(23)}=1.97, p=0.06\right)$. There was no effect of current SRI medication $(p>0.2)$.

\section{Evoked Cardiac Response}

The group (panic disorder $v s$ major depression $v s$ control) $\times$ valence ANOVA on the peak cardiac acceleration revealed no main effects or interactions (all $p$-values $>0.2$, Figure 1 ).

\section{Independent Effects of PD on P300 and N300H}

In comparison to controls, participants with PD showed larger $\mathrm{N} 300 \mathrm{H}$ when controlling for P300 amplitude $\left(t_{(43)}=2.28, p<0.03\right)$ or individual cross-trial standard deviations in EEG magnitude at the $\mathrm{N} 300 \mathrm{H}$ latency $\left(\mathrm{t}_{(43)}=2.29, \mathrm{p}<0.03\right)$. Conversely, participants with PD $v s$ controls also showed larger P300 amplitude when controlling for $\left.\mathrm{N} 300 \mathrm{H}\left(t_{(43)}=2.07\right), p<0.05\right)$. These results indicate that the two effects reflect independent characteristics of PD in the present sample.

\section{DISCUSSION}

Interactions of higher cortical processes and visceral, especially cardiac activity have been assumed to have an important role in panic attacks and PD (Bouton et al, 2001; Clark, 1986; Friedman and Thayer, 1998; Gorman et al, 2000). Employing the novel CECT method to assess corticocardiac coupling, this study demonstrates for the first time that brain-heart covariation is significantly elevated in individuals suffering from PD. Converging with prior evidence for serotonergic involvement in the cortical control of the heart, it was further shown that current SRI treatment predicted increased cortico-cardiac coupling. 
Importantly, PD was associated with potentiated coupling even in non-medicated individuals, thereby demonstrating that concurrent serotonergic medication alone does not explain elevated brain-heart covariation in PD.

Brain-heart coupling was assessed with the CECT feature $\mathrm{N} 300 \mathrm{H}$, which reflects the strongest within-subject correlation of EEG at 250-500 ms after a feedback stimulus and subsequent accelerations of heart rate. The estimated source of $\mathrm{N} 300 \mathrm{H}$ is the anterior cingulate cortex (ACC; Panitz et al, 2013) a critical cortical structure for central control of the viscera (Critchley et al, 2003), which indirectly projects to the nucleus ambiguous where cell bodies of vagal preganglionic neurons are located (Thayer and Lane, 2009). Because the time-frequency characteristics of $\mathrm{N} 300 \mathrm{H}$ indeed suggest underlying vagal processes (Mueller et al, 2010) the $\mathrm{N} 300 \mathrm{H}$ may result from an efferent pathway in which the ACC/medial prefrontal cortex triggers relative cardiac acceleration via vagal dis-inhibition (Mueller et al, 2013; Thayer and Lane, 2000). Alternatively, N300H-like phenomena could also emerge if other brain regions trigger visceral reactions to the feedback stimulus and send parallel signals to the cortex (ie, efferent copies) where they may alter the EEG signal at $300 \mathrm{~ms}$ (Nieuwenhuis et al, 2011).

Accordingly, one interpretation of the potentiated $\mathrm{N} 300 \mathrm{H}$ in $\mathrm{PD}$ is a hyperreactive cortico-vagal pathway in these individuals, potentially leading to excessive autonomic lability (Eysenck, 1970) while contributing to low tonic vagal activity due to frequent vagal withdrawal (Friedman et al, 1998). However, the accelerative response in the evoked (ie, trial averaged) HP was not very strong, and for some subjects the greatest acceleration may have been a reduced deceleration. Furthermore, there were no significant group differences in evoked HP, although the PD group tended to show the maximum accelerative response. The lack of strong effects on evoked HP may be explained by further influences on evoked HP, which blur pure corticovagal effects (eg, sympathetic influences, baroreceptor reflexes, etc). Alternatively, however, PD may be characterized by a potentiated efferent copy (leading to potentiated cortico-cardiac covariation) rather than the cardiac response itself. This alternative interpretation is supported by (A) an absence of significant group differences in the feedback-evoked cardiac response, (B) significantly increased P300 in panic disorder (see also: Clark et al, 1996), which may indicate an efferent copy mechanism (Nieuwenhuis et al, 2011) and (C) prior reports of increased cardiac awareness in PD (Ehlers and Breuer, 1992).

Irrespective of the precise underlying mechanism, excessive neurovisceral covariation may predispose individuals with PD to experiencing sudden physiological reactions more often than non-anxious individuals. The experience of such neurogenic physiological changes may contribute to panic attacks, particularly when negatively interpreted as suggested by prominent models (Clark, 1986; McNally, 1990). However, a direct link between $\mathrm{N} 300 \mathrm{H}$ magnitude and panic attacks remains to be demonstrated in future studies, in order to further clarify how increased brain-heart covariation relates to $\mathrm{PD}$.

In addition to $\mathrm{N} 300 \mathrm{H}$ magnitude, $\mathrm{PD}$ was associated with an extended temporal spread of brain-heart covariation. Thus, the brain signal that was associated with subsequent cardiac modulation was not only more correlated with HP but also detectable within a larger time frame than in participants without history of panic (ie, MD and control group). Possibly, ANS-related brain activity reverberates longer in $\mathrm{PD}$, thereby more persistently enforcing neurogenic vagal modulations in these individuals and/or yielding more salient cortical representations of autonomic reactions, which could further contribute to panic attacks. Although the interpretation of this exploratory analysis is necessarily preliminary, it provides additional evidence for abnormal brain-heart coupling in PD.

In contrast to $\mathrm{PD}, \mathrm{MD}$ individuals did not show higher $\mathrm{N} 300 \mathrm{H}$ magnitude than the control group and showed significantly lower temporal spread of $\mathrm{N} 300 \mathrm{H}$ than the PD group. The overall pattern of mean $\mathrm{N} 300 \mathrm{H}$ and $\mathrm{P} 300$ amplitude across groups (control $<\mathrm{MD}<\mathrm{PD}$ ), however, closely mirrors the pattern of panic-related questionnaire scores (ASI, ACQ) and thus provides further support for the previously discussed association between $\mathrm{N} 300 \mathrm{H}$ and panic. Although individuals with MD reported no history of panic attacks, MD increases the risk for developing PD in the future (Hayward et al, 2000). Moreover, MD has been associated with cortico-vagal abnormalities as indicated by heart rate variability (Carney et al, 2001), making depression an interesting candidate for future CECT studies. Future studies with larger sample sizes may test whether there is a robust elevation of $\mathrm{N} 300 \mathrm{H}$ in $\mathrm{MD}$.

Of relevance, $\mathrm{PD}$ and $\mathrm{MD}$ participants currently taking SRIs displayed higher $\mathrm{N} 300 \mathrm{H}$ compared with other participants with PD or MD. Because by blocking the reuptake of $5-\mathrm{HT}$, SRIs induce an increase in extrasynaptic 5-HT availability, the potentially SRI-induced elevation of $\mathrm{N} 300 \mathrm{H}$ converges with (a) the general involvement of 5HT in central control of the heart (Jordan, 2005) and (b) prior findings of a positive relationship between 5-HT and $\mathrm{N} 300 \mathrm{H}$ (Mueller et al, 2012; Mueller et al, 2013). Moreover, the effect of SRIs on brain-heart coupling may explain altered heart rate variability in humans with long-term SRI treatments (Licht et al, 2010) and potentiated cardiovascular responses to acute stress in rats with chronic fluoxetine administration (Grippo et al, 2006).

It should be noted that medication was not related to lower questionnaire symptom scores in the present sample. Thus, it would be incorrect to assume that individuals who are taking SRIs are characterized by reduced symptoms and should therefore also have reduced N300H. Moreover, consistent with the wide-spread localization of 5-HT signaling in the human brain (Cools et al, 2008), SRIs are known to have both anxiolytic and anxiogenic effects, with the anxiogenic effects being more pronounced at the beginning of treatment (Goldstein and Goodnick, 1998; Stahl, 1998; Trindade et al, 1998) often causing treatment discontinuation (Zanardi et al, 1996). In light of the positive relationship between anxiety and $\mathrm{N} 300 \mathrm{H}$ (Mueller et al, 2013), we hypothesize that SRIs by increasing brain-heart coupling produce anxiety-like side effects, which manifest at the beginning of treatment when anxiolytic effects of SRIs are not yet established (Stahl, 1998). However, this suggestion remains to be tested given that the length of time on medication and response to SRI treatment were not assessed in the present study. Moreover, because pharmacological treatment was not experimentally manipulated, there may be systematic differences in the two groups that 
drive the observed effects (eg, more severe forms of PD/MD in the SRI $v s$ non-medicated group). Thus, future longitudinal and experimental CECT studies should test whether brain-heart covariation mediates short and long-term effects of SRIs on panic and anxiety.

In conclusion, PD and SRIs were associated with elevated covariation of brain and heart activity using the novel CECT method in a gambling task. This might indicate that PD is characterized by a general (ie, stimulus independent) abnormality of brain-heart coupling, which may relate to 5-HT and stimulus-independent unexpected panic attacks. However, future work will have to test (1) whether the present findings generalize to other tasks and stimuli, (2) whether potentiated brain-heart coupling directly facilitates panic attacks, for example, by increasing neurogenic vagal withdrawal during panic and/or the cortical processing of neurovisceral efferences, and (3) how serotonin and SRIs affect such mechanisms.

\section{FUNDING AND DISCLOSURE}

The authors declare no conflict of interest.

\section{ACKNOWLEDGEMENTS}

This work was funded by Deutsche Forschungsgemeinschaft grant number DFG WA 2593/2-2.

\section{REFERENCES}

Aronson TA, Carasiti I, McBane D, Whitaker-Azmitia P (1989). Biological correlates of lactate sensitivity in panic disorder. Biol Psychiatry 26: 463-477.

Barlow DH, Vermilyea J, Blanchard EB, Vermilyea BB, Di Nardo PA, Cerny JA (1985). The phenomenon of panic. J Abnorm Psychol 94: 320-328.

Beck AT, Steer RA, Ball R, Ranieri W (1996). Comparison of Beck Depression Inventories -IA and -II in psychiatric outpatients. J Pers Assess 67: 588-597.

Bouton ME, Mineka S, Barlow DH (2001). A modern learning theory perspective on the etiology of panic disorder. Psychol Rev 108: 4-32.

Carney RM, Blumenthal JA, Stein PK, Watkins L, Catellier D, Berkman LF et al (2001). Depression, heart rate variability, and acute myocardial infarction. Circulation 104: 2024-2028.

Chambless DL, Caputo GC, Bright P, Gallagher R (1984). Assessment of fear of fear in agoraphobics: the body sensations questionnaire and the agoraphobic cognitions questionnaire. J Consult Clin Psychol 52: 1090-1097.

Clark CR, McFarlane AC, Weber DL, Battersby M (1996). Enlarged frontal P300 to stimulus change in panic disorder. Biol Psychiatry 39: 845-856.

Clark DM (1986). A cognitive approach to panic. Behav Res Ther 24: 461-470.

Cools R, Roberts AC, Robbins TW (2008). Serotoninergic regulation of emotional and behavioural control processes. Trends Cogn Sci 12: 31-40.

Cowley DS, Hyde TS, Dager SR, Dunner DL (1987). Lactate infusions: the role of baseline anxiety. Psychiatry Res 21: 169-179.

Critchley HD, Mathias CJ, Josephs O, O’Doherty J, Zanini S, Dewar BK et al (2003). Human cingulate cortex and autonomic control: converging neuroimaging and clinical evidence. Brain 126: 2139-2152.
David SP, Murthy NV, Rabiner EA, Munafo MR, Johnstone EC, Jacob $\mathrm{R}$ et al (2005). A functional genetic variation of the serotonin (5-HT) transporter affects 5-HT1A receptor binding in humans. J Neurosci 25: 2586-2590.

Delorme A, Makeig S (2004). EEGLAB: an open source toolbox for analysis of single-trial EEG dynamics including independent component analysis. J Neurosci Methods 134: 9-21.

Ehlers A, Breuer P (1992). Increased cardiac awareness in panic disorder. J Abnorm Psychol 101: 371-382.

Eysenck HJ (1970). The Structure of Human Personality. Meuthen \& Co: London, UK.

Friedman BH, Thayer JF (1998). Autonomic balance revisited: panic anxiety and heart rate variability. J Psychosom Res 44: 133-151.

Gaffney FA, Fenton BJ, Lane LD, Lake CR (1988). Hemodynamic, ventilatory, and biochemical responses of panic patients and normal controls with sodium lactate infusion and spontaneous panic attacks. Arch Gen Psychiatry 45: 53-60.

Goldstein BJ, Goodnick PJ (1998). Selective serotonin reuptake inhibitors in the treatment of affective disorders-III. Tolerability, safety and pharmacoeconomics. J Psychopharmacol 12: S55-S87.

Gorman JM, Kent JM, Sullivan GM, Coplan JD (2000). Neuroanatomical hypothesis of panic disorder, revised. Am J Psychiatry 157: 493-505.

Graeff FG, Del-Ben CM (2008). Neurobiology of panic disorder: from animal models to brain neuroimaging. Neurosci Biobehav Rev 32: 1326-1335.

Grippo AJ, Beltz TG, Weiss RM, Johnson AK (2006). The effects of chronic fluoxetine treatment on chronic mild stress-induced cardiovascular changes and anhedonia. Biol Psychiatry 59: 309-316.

Hayward C, Killen JD, Kraemer HC, Taylor CB (2000). Predictors of panic attacks in adolescents. J Am Acad Child Adolesc Psychiatry 39: 207-214.

Jordan D (2005). Vagal control of the heart: central serotonergic (5-HT) mechanisms. Exp Physiol 90: 175-181.

Kemper CJ (2010). Das Persönlichkeitsmerkmal Angstsensitivität: Taxon oder Dimension?. Verlag Dr Kovač: Hamburg, Germany.

Kendler KS, Gardner CO, Prescott CA (2001). Panic syndromes in a population-based sample of male and female twins. Psychol Med 31: 989-1000.

Licht CM, de Geus EJ, van Dyck R, Penninx BW (2010). Longitudinal evidence for unfavorable effects of antidepressants on heart rate variability. Biol Psychiatry 68: 861-868.

Margraf J (1990). Ambulatory psychophysiological monitoring of panic attacks. 1990. J Psychophysiol 4: 321-330.

Margraf J (1994). Mini-DIPS: Diagnostisches Kurz-Interview bei psychischen Störungen. Springer: Berlin (Germany).

McNally RJ (1990). Psychological approaches to panic disorder: a review. Psychol Bull 108: 403-419.

Mueller EM, Evers EA, Wacker J, van der Veen FM (2012). Acute tryptophan depletion attenuates brain-heart coupling following external feedback. Front Hum Neurosci 6: 77.

Mueller EM, Makeig S, Stemmler G, Hennig J, Wacker J (2011). Dopamine effects on human error processing depend on catechol-o-methyltransferase VAL158MET genotype. J Neurosci 31: $15818-15825$.

Mueller EM, Stemmler G, Hennig J, Wacker J (2013). 5-HTTLPR and anxiety modulate brain-heart covariation. Psychophysiology 50: 441-453.

Mueller EM, Stemmler G, Wacker J (2010). Single-trial electroencephalogram predicts cardiac acceleration: a time-lagged P-correlation approach for studying neurovisceral connectivity. Neuroscience 166: 491-500.

Neumeister A, Bain E, Nugent AC, Carson RE, Bonne O, Luckenbaugh DA et al (2004). Reduced serotonin type 1A receptor binding in panic disorder. J Neurosci 24: 589-591. 
Nieuwenhuis S, De Geus EJ, Aston-Jones G (2011). The anatomical and functional relationship between the P3 and autonomic components of the orienting response. Psychophysiology 48: $162-175$.

Panitz C, Wacker J, Stemmler G, Mueller EM (2013). Brain-heart coupling at the P300 latency is linked to anterior cingulate cortex and insula-a cardio-electroencephalographic covariance tracing study. Biol Psychol 94: 185-191.

Roy-Byrne PP, Stang P, Wittchen HU, Ustun B, Walters EE, Kessler RC (2000). Lifetime panic-depression comorbidity in the National Comorbidity Survey. Association with symptoms, impairment, course and help-seeking. $\mathrm{Br} J$ Psychiatry 176: 229-235.

Sato A, Yasuda A, Ohira H, Miyawaki K, Nishikawa M, Kumano H et al (2005). Effects of value and reward magnitude on feedback negativity and P300. Neuroreport 16: 407-411.
Stahl SM (1998). Mechanism of action of serotonin selective reuptake inhibitors. Serotonin receptors and pathways mediate therapeutic effects and side effects. J Affect Disord 51: 215-235.

Thayer JF, Lane RD (2000). A model of neurovisceral integration in emotion regulation and dysregulation. J Affect Disord 61: 201-216.

Thayer JF, Lane RD (2009). Claude Bernard and the heart-brain connection: further elaboration of a model of neurovisceral integration. Neurosci Biobehav Rev 33: 81-88.

Trindade E, Menon D, Topfer LA, Coloma C (1998). Adverse effects associated with selective serotonin reuptake inhibitors and tricyclic antidepressants: a meta-analysis. CMAJ 159: 1245-1252.

Zanardi R, Franchini L, Gasperini M, Perez J, Smeraldi E (1996). Double-blind controlled trial of sertraline versus paroxetine in the treatment of delusional depression. Am J Psychiatry 153: 1631-1633. 